\title{
Involvement of CD28/CTLA4-B7 Costimulatory Pathway in the Development of Lymphadenopathy and Splenomegaly in MRL/lpr Mice
}

\author{
Mitsuyoshi TAKIGUCHI, Masaaki MURAKAMI ${ }^{1)}$, Izumi NAKAGAWA ${ }^{1)}$, Mohammod Misanur RASHID ${ }^{1)}$, \\ Noriko TOSA $^{1)}$, Shunsuke CHIKUMA ${ }^{1)}$, Akira HASHIMOTO and Toshimitsu UEDE ${ }^{1 *}$ \\ Laboratory of Pathobiology, Department of Veterinary Clinical Sciences, Graduate School of Veterinary Medicine, Hokkaido \\ University, Sapporo 060-0818 amd ${ }^{1)}$ Section of Immunopathogenesis, Institute of Immunological Science, Hokkaido University, Sapporo \\ 060-0815, Japan
}

(Received 23 July 1999/Accepted 3 September 1999)

ABSTRACT. MRL/lpr mouse is an established animal model which develops autoimmune diseases including glomerulonephritis, sialoadenitis, hepatitis and inflammatory lung disease. Additionally, it has been reported that $l p r$ strains uniquely accumulate $\mathrm{CD} 3^{+} \mathrm{CD} 4{ }^{-} \mathrm{CD} 8^{-} \mathrm{B} 220^{+}$ (double negative, DN) T cells in lymphoid organs leading to lymphadenopathy and splenomegaly. To investigate the role of CD28/ CTLA4-B7 pathway in the development of lymphadenopathy and splenomegaly, MRL/lpr mice were treated with soluble form of CTLA4 molecules, CTLA4IgG, which efficiently blocks this pathway. It was demonstrated that (i) the development of DN T cells was independent of the CD28/CTLA4-B7 pathway, (ii) the CD28/CTLA4-B7 pathway was required for the development of lymphadenopathy and splenomegaly, (iii) the CD28/CTLA4-B7 pathway was important for the accumulation of various cell populations in the lymph node and spleen, (iv) composition of the accumulating cell populations was not altered by CTLA4IgG treatment, and (v) activation of conventional T cells and IL-4 production from conventional T cells were the CD28/CTLA4-B7 pathway dependent. Thus, we concluded that the CD28/ CTLA4-B7 pathway was required for the development of full-blown lymphadenopathy and splenomegaly in MRL/lpr mice.-KEY wORDS: costimulatory signal, CTLA4IgG, IL-4, lymphadenopathy, MRL/lpr.

J. Vet. Med. Sci. 62(1): 29-36, 2000

MRL/Mp-lpr/lpr (MRL/lpr) mice develop progressive nonmalignant lymphoproliferative disease characterized by splenomegaly, severe lymphadenopathy, hypergammaglobulinemia, circulating autoantibodies, and premature death $[3,10]$. A single autosomal recessive gene, termed lymphoaccumulation (lpr) represents a mutation, a nearly disabling insertion of an early retrotransposon in the Fas apoptosis gene [1, 7, 23, 47-49], causes defective activation-induced death of hematopoietic cells including $\mathrm{T}$, B cells, macrophages and so on $[12,38,40,46]$. Lymphadenopathy and splenomegaly result largely from the progressive accumulation of unusual population of thymusderived $\mathrm{B} 220^{+}$, T cell receptor (TCR) $\alpha \beta^{+}, \mathrm{CD}^{+}, \mathrm{CD} 44^{+}$, $\mathrm{CD} 28^{+}, \mathrm{CD} 2^{-}, \mathrm{CD}^{-}, \mathrm{CD} 8^{-} \mathrm{T}(\mathrm{DN} \mathrm{T})$ cells that spontaneously proliferated in vivo [52] and expressed several cytokines including interferon (IFN)- $\gamma$, tumor necrosis factor (TNF)- $\alpha$, and early T lymphocyte activation protein 1 (Eta1) $[29,33,34]$. DN T cells are first detectable within 1 month of age and represent more than $70 \%$ of lymph node cells by 20 weeks of age [43]. It is well established that DN T cells develop from thymic precursors [24, 32], but the lineage of immediate precursors of DN T cells was controversial. Recently, it has been demonstrated that majority of DN $\mathrm{T}$ cells are derived from major histocompatibility complex (MHC) class I-restricted CD8 ${ }^{+}$

* Correspondence to: Dr. Uede, T., Section of Immunopathogenesis, Institute of Immunological Science, Hokkaido University, Kita-15, Nishi-7, Kita-ku, Sapporo 060-0815, Japan. precursors [15]. In addition to this predominant DN T cells, spleen and lymph nodes of MRL/lpr mice contain a minor subset of $\mathrm{B} 220^{+} \mathrm{CD} 4^{+} \mathrm{T}$ cells that resembles the DN T cells in ontogeny and phenotype $[4,21]$. Additionally, absolute numbers of $\mathrm{T}, \mathrm{B}$ cells, and macrophages significantly increase. However, the mechanisms leading to lymphadenopathy and splenomegaly are not fully understood.

$\mathrm{T}$ cells require two signals for full activation $[19,26]$. The first signal is provided by ligation of the TCR with MHC molecule plus the antigenic peptide on antigen presenting cells (APC), and thus provides antigen specificity to the immune responses. The second costimulatory signal is provided by binding of specific receptors on $\mathrm{T}$ cells with their ligands on APC [20, 28]. The best-characterized costimulatory pathway is provided by CD28 molecules on T cells interacting with B7 family molecules on APC [17, 18, 31]. Cytotoxic T lymphocyte-associated antigen 4 (CTLA4), a homologue of CD28 is found on activated T cells but not on resting ones and transduces negative costimulatory signal in vitro and in vivo [22, 25, 27]. CTLA4 binds to B7 or related molecules on APC with higher avidity than CD28 does. Blockade of CD28-B7 pathway using CTLA4IgG inhibits autoimmune and alloimmune responses and in some cases induces $\mathrm{T}$ cell anergy or antigen specific tolerance in vitro and in vivo $[2$, $6,11,41,42]$. We speculated that if DN T cells arise constantly from Fas defective $\mathrm{T}$ cells and/or these cells are able to proliferate in vivo in response to certain stimulations such as soluble factors and adhesion molecules, they may 
use CD28/CTLA4-B7 pathway as a costimulatory signal. Thus, we investigated the role of the CD28/CTLA4-B7 costimulatory pathway in the development of lymphadenopathy and splenomegaly. It was demonstrated that the CD28/CTLA4-B7 pathway was important for the development of lymphadenopathy and splenomegaly. We also demonstrated that the accumulation of all cell populations including abnormal $\mathrm{DN} \mathrm{T}, \mathrm{CD} 4{ }^{+} \mathrm{B} 220^{+} \mathrm{T}$ cells, conventional T, B cells, and macrophages was the CD28/ CTLA4-B7 pathway dependent. Additionally, the role of interleukin (IL)-4 molecules in induction of lymphadenopathy and splenomegaly was discussed.

\section{MATERIALS AND METHODS}

Mice: MRL/lpr mice were purchased from Japan SLC Co. (Shizuoka, Japan). Mice were housed under specific pathogen-free condition in the animal facility at the Institute of Immunological Science, Hokkaido University. Mice used in this study were approximately 3 weeks old or within 24 $\mathrm{hr}$ after birth at initiation of the treatment regimen, with either CTLA4IgG consisting of an extracellular region of mouse CTLA4 and a Fc portion of human IgG1 or with human $\operatorname{IgG}(\operatorname{IgG})$ as a control.

Treatment procedure: Mouse CTLA4IgG was generated from Adex1CACTLA4IgG [35] and purified as previously described [31]. Mice were injected intraperitoneally three times a week with $100 \mu \mathrm{g} / \mathrm{head}$ of either CTLA4IgG or IgG starting at 3 weeks of age or within $24 \mathrm{hr}$ after birth until 20 weeks of age.

Score of lymphadenopathy: The lymphadenopathy was evaluated as scores 0 to 3 as previously described [51]. The diameter of lymph nodes including left and right mandibular, axillary, and inguinal lymph nodes was measured and graded as follows: $0 ; 0-5 \mathrm{~mm}, 1 ; 6-10 \mathrm{~mm}, 2 ; 11-15 \mathrm{~mm}, 3 ; 16-$ $20 \mathrm{~mm}$. The total score of each mouse was the summation of the individual score of the lymph nodes.

Antibodies: Phycoerythrin (PE)-anti-mouse IL-4 (rat monoclonal antibody (mAb), 11B11); PE-anti-mouse IFN- $\gamma$ (rat mAb, XMG1.2); PE-rat IgG1 (rat mAb, R3-34); fluorescein isothiocyanate (FITC)-anti-mouse CD3 (hamster mAb, 145-2C11); FITC-, PE-, and biotin-anti-mouse CD4 (rat mAb, H129.19); FITC-, PE-, and biotin-anti-mouse B220 (rat mAb, RA3-6B2); PE-anti-mouse CD69 (rat mAb, H1.2F3); PE-anti-mouse B7-1 (rat mAb, 16-10A1); PEanti-mouse B7-2 (rat mAb, GL1); and PE- and biotin-anti-mouse CD8 (rat mAb, 53-6.7); and anti-mouse $\mathrm{Fc} \gamma$ receptor $(\mathrm{Fc} \gamma \mathrm{R})$ II and III (rat $\mathrm{mAb}, 2.4 \mathrm{G} 2$ ) were purchased from PharMingen (San Diego, U.S.A.). PE-antimouse CD71 (rat mAb, RI7217.1.4) and FITC-anti-mouse Mac1 (rat mAb, M1/70.15) were purchased from Caltag Lab. (Burlingame, U.S.A.). Red670-avidin was purchased from Gibco (Gaithersburg, U.S.A.).

Flow cytometric analysis: Peripheral nonmesenteric lymph nodes and spleen were homogenized and cleared of erythrocytes by osmotic lysis. For surface-immunostaining, cells $\left(5 \times 10^{5}\right)$ were pretreated with anti-Fc $\gamma \mathrm{RII}$ and III $\mathrm{mAb}$,
2.4G2 for $1 \mathrm{hr}$ and washed. Then cells were incubated with $\mathrm{mAb}$ in PBS containing $1 \%$ bovine serum albumin (BSA) and $0.05 \% \mathrm{NaN}_{3}$, washed and incubated with second step reagents, avidin-Red670 for $30 \mathrm{~min}$. In some experiments, propidium iodide (Sigma, St. Louis, U.S.A.) was added for last 1 min. Living cells were given a final wash and analyzed on a FACScan (Becton Dickinson, San Jose, U.S.A.). For intracellular-staining, cells $\left(1 \times 10^{6}\right)$ were first stained with antibodies against cell surface molecules described above and then permealyzed by FACS permealyzing solution (Becton Dickinson, San Jose, U.S.A.) for $15 \mathrm{~min}$ at room temperature. Then, intracellular cytokines were stained with PE-conjugated anti-cytokine $\mathrm{mAbs}$ for $30 \mathrm{~min}$ at room temperature. Cells were given a final wash, resuspended in $1 \%$ paraformaldehide in PBS, and analyzed on a FACScan.

Statistical analysis: Statistical significance was determined by using the Mann-Whitney $U$ test.

\section{RESULTS}

Lymphadenopathy and splenomegaly in CTLA4IgGtreated mice: In control animals $(\mathrm{n}=15), l p r$-induced lymphadenopathy as determined by diameter of lymph nodes was increased at 12 to 20 weeks of age (Fig. 1). On the other hand, in mice treated with CTLA4IgG from day 21 $(n=15)$, score of lymph node diameter was significantly reduced as compared with that of IgG treated-MRL/lpr mice (Fig. 1). Since CTLA4IgG treatment from day $21 \mathrm{did}$ not dissolve lymphadenopathy completely, we treated two groups ( $\mathrm{n}=15 \mathrm{each}$ ) of neonatal (within $24 \mathrm{hr}$ after birth) female MRL/lpr mice with either CTLA4IgG or IgG, 100 $\mu \mathrm{g}$, intraperitoneally three times per week for 20 weeks. Animals treated with CTLA4IgG from day 0 exhibited significant reduction of lymph node diameter (Fig. 1). At 20 to 22 weeks mice were sacrificed and analyzed for organ weight of the lymph node and spleen. As shown in Fig. 2, after CTLA4IgG treatment, organ weight of the lymph node and spleen was significantly reduced as compared with that of control mice treated with IgG; approximate organ weight of $1.2 \mathrm{~g}$ versus $0.17 \mathrm{~g}$ in the lymph node and $0.8 \mathrm{~g}$ versus $0.3 \mathrm{~g}$ in the spleen (IgG versus CTLA4IgG treatment, respectively). Thus, in MRL/lpr mice, the CD28/CTLA4B7 signaling pathway was involved in the development of lymphadenopathy and splenomegaly.

Composition of accumulating cells in CTLA4IgG-treated mice: As can be expected from the data shown in Figs. 1 and 2, mice treated with CTLA4IgG from day 0 exhibited significant reduction of actual number of accumulating lymphoid cells in the lymph node and spleen (Fig. 3A). Approximate cell numbers are $8.7 \times 10^{8}$ and $1.1 \times 10^{8}$ in the lymph node and $2.9 \times 10^{8}$ and $1.1 \times 10^{8}$ in the spleen in control and CTLA4IgG-treated mice, respectively. We then carefully examined the percentage of various cell populations. In agreement with previous reports [43], there is an accumulation of a large number of abnormal $\mathrm{T}$ cells in the lymph node and spleen. Abnormal DN T cells represent 


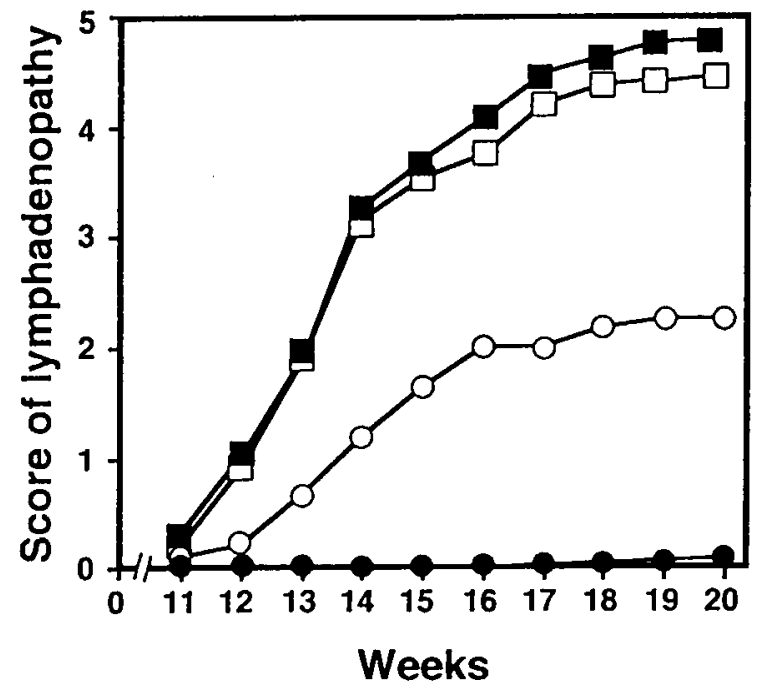

Fig. 1. Lymphadenopathy in MRL/lpr mice. Lymphadenopathy was evaluated by measuring diameter of six lymph nodes such as left and right mandibular, axillary and inguinal lymph nodes and graded as follows: $0,0-5 \mathrm{~mm} ; 1,6-10 \mathrm{~mm} ; 2,11-15 \mathrm{~mm} ; 3,16-20 \mathrm{~mm}$. The total score of each mouse was the summation of the individual score of six lymph nodes. Mice treated with CTLA4IgG $(\bigcirc)$ or $\operatorname{IgG}(\square)$ from day 21 and with CTLA4IgG (O) or IgG (ם) from day 0. Each group consisted of 15 mice. Data represent the mean.

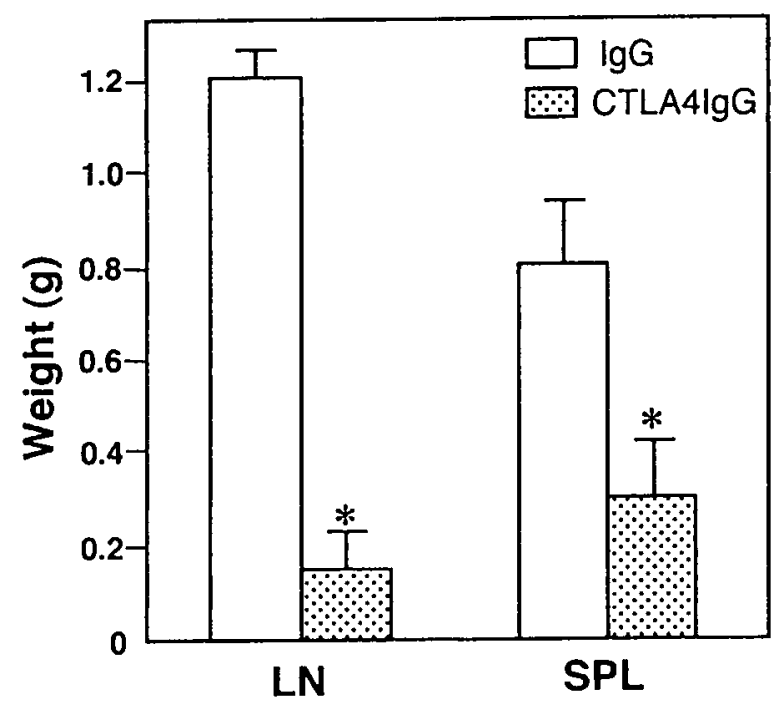

Fig. 2. Organ weight of the lymph nodes and spleen. Six peripheral nonmesenteric lymph nodes (LN) and spleen (SPL) were isolated at 20 to 22 weeks from mice treated with either CTLA4IgG or IgG from birth and were analyzed for weight. Each group consisted of 6 mice. Data represent the mean \pm SD. The significance of differences in both groups was estimated by using the Mann-Whitney $U$ test. $* \mathrm{P}<0.001$.
73 and $19 \%$ of total cell population in the lymph node and spleen of control mice, respectively (Fig. 3B). While, in CTLA4IgG-treated mice, DN T cells are $68 \%$ and $25 \%$ in the lymph node and spleen, respectively. B220 ${ }^{+} \mathrm{B}$ cells are 10 to $13 \%$ in the lymph node and $30 \%$ in the spleen. CD $4^{+}$ $\mathrm{T}$ cells are $4 \%$ in the lymph node and 14 to $16 \%$ in the spleen of both control and CTLA4IgG-treated mice, respectively. There are very few $\mathrm{CD}^{-}{ }^{-} \mathrm{B} 220^{-}$cells in the lymph node, while there are 14 to $18 \%$ of those cells in the spleen of both control and CTLA4IgG-treated mice, respectively. Thus, it is noteworthy that CTLA4IgG-treated mice maintained similar profile of cellular subsets in the both lymph node and spleen as compared with control $l p r$ mice.

Inhibition of IL-4 production by conventional $T$ cells in CTLA4IgG-treated mice: We observed that lymphadenopathy and splenomegaly were significantly reduced by CTLA4IgG treatment, but the composition of accumulating cells in lymphoid organs was not altered (Figs. 1 to 3). These phenotypes were very similar to those of IL4-deficient MRL/lpr mice [36]. Therefore, we hypothesized that production of IL-4 from certain cell populations was inhibited after CTLA4IgG treatment. It has been reported that activated T cells isolated from lymph nodes of MRL/ $l p r$ mice expressed significant amount of IL-4 mRNA [5, 44]. Conventional CD4 ${ }^{+} \mathrm{T}$ cells in lymph nodes from MRL/ lpr mice treated with CTLA4IgG or IgG were evaluated for expression of activation markers and intracellular cytokines such as IL-4 and IFN- $\gamma$ molecules (Fig. 4). CD4 ${ }^{+} \mathrm{T}$ cells in control MRL/lpr mice expressed significant level of activation markers such as B7-1, B7-2 and CD69 and intracellular cytokine IL-4, but not IFN $\gamma$ (Fig. 4). In contrast, CD4 ${ }^{+} \mathrm{T}$ cells derived from CTLA4IgG-treated animals expressed reduced amounts of activation markers and intracellular IL-4 (Fig. 4). Similar results were obtained from $\mathrm{CD}^{+} \mathrm{T}$ cells (data not shown). Thus, administration of CTLA4IgG prevented activation and IL-4 production of conventional T cells efficiently in MRL/lpr mice.

Finally we examined whether the inhibition of cytokine production by CTLA4IgG is IL-4 specific. Thus, we analyzed the intracellular cytokine production by DN T cells and macrophages. In contrast to conventional $\mathrm{CD} 4^{+} \mathrm{T}$ cells, the $\mathrm{CD}^{+}{ }^{+} \mathrm{CD} 4^{-} \mathrm{CD} 8^{-}$fraction, which also express $\mathrm{B} 220$ molecules in control mice produced IFN- $\gamma$, but not IL-4 (Fig. 5). This intracellular IFN $\gamma$ production was significantly inhibited by CTLA4IgG treatment (Fig. 5). However, expression of activation markers and intracellular cytokine such as B7-1, B7-2, CD71 and IFN $\gamma$ in the CD3-B220fraction was not inhibited by CTLA4IgG treatment (Fig. 6). Because there were few NK cells as determined by flow cytometer by using anti-NK cell mAb, DX-5 (within $3 \%$ in the $\mathrm{CD}^{-}{ }^{-} \mathrm{B} 220^{-}$fraction, data not shown), the CD3-B220fraction mainly consisted of $\mathrm{Mac}^{+}$macrophages was activated and produced INF- $\gamma$ in the CD28/CTLA4-B7 pathway independent manner. 

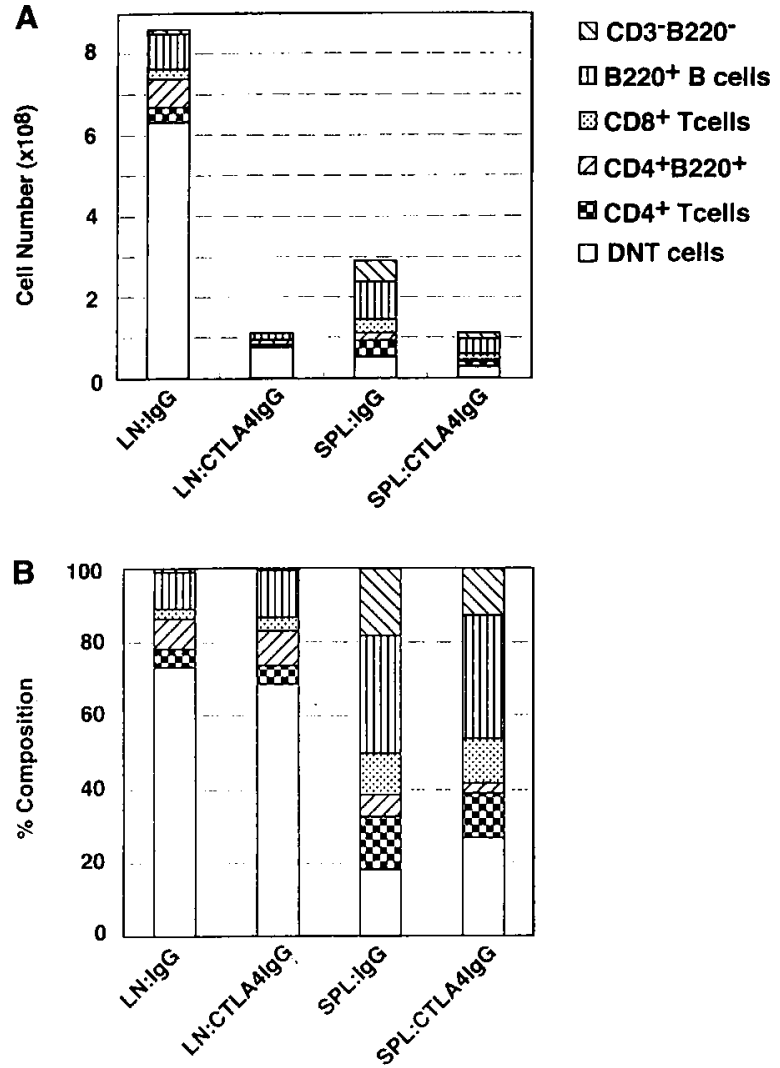

Fig. 3. Cell number and a profile of cellular subsets. Six peripheral nonmesenteric lymph nodes (LN) and spleen (SPL) were isolated at 20 to 22 weeks from mice treated with either CTLA4IgG or IgG from birth and these cells were analyzed by using FACS. (A) and (B) indicated cell number and percentage of individual cell population, respectively. Each group consisted of 6 mice. Data represent the mean.

\section{DISCUSSION}

This study was performed to examine the role of the CD28/CTLA4-B 7 costimulatory pathway in the development of lymphadenopathy and splenomegaly in the MRL/lpr strain. It has been shown that lymphadenopathy and splenomegaly in MRL/lpr mice result largely from the progressive accumulation of DN T cells and to some extent, various lymphoid cell populations [9]. The mechanism leading to accumulation of various cell populations are clearly dependent on Fas abnormality, which causes the impairment of activation-induced cell death [30]. In fact, transgenic expression of Fas molecules on $\mathrm{T}$ cells in $l p r$ mice resulted in the reduction of the lymphadenopathy [13, 50]. It was reported that the origin of majority of DN T cells was thymus-derived class I-restricted $\mathrm{CD} 8^{+} \mathrm{T}$ cells [15]. It was recently shown that limited but certain proliferation of DN T cells was induced after anti-TCR plus anti-CD28 mAb in vitro $[8,16]$. These results brought us a speculation that if DN T cells arise constantly from stimulated $\mathrm{CD} 8^{+} \mathrm{T}$ cells and/or these cells are able to proliferate in vivo in response to a certain stimulation, they may use the CD28/CTLA4-B7 pathway as a costimulatory signal. At first, we injected CTLA4IgG from 3 weeks of age, but this treatment could not meet with a complete ameriolation of lymphadenopathy and splenomegaly (Fig. 1). Treatment with CTLA4IgG from day 0 after birth resulted in almost complete suppression of lymphadenopathy and splenomegaly (Fig. 1 and data not shown), but there were still significant number of DN T cells in the lymph node and spleen (Fig. 3). Therefore, these results indicated that (i) the development of DN T cells was independent of the CD28/CTLA4-B7 pathway, (ii) the CD28/CTLA4-B7 pathway was required for the development of lymphadenopathy and splenomegaly. Thus, the CD28/ CTLA4-B7 signaling pathway was important for the accumulation of various cell populations in the lymph node and spleen.

It became clear that cytokines also played a critical role for the development of lymphadenopathy and splenomegaly. Recently, IL-4-deficient $l p r$ mice were generated and exhibited significant amelioration of lymphadenopathy and splenomegaly [36]. More importantly, IL-4-deficient $l p r$ mice maintained similar profile of cellular subsets as compared with control lpr-animals [36]. This phenotype was very similar to what we found in CTLA4IgG-injected lpr mice. In addition, in CTLA4IgG-treated $l p r$ mice, activation of $\mathrm{CD}^{+} \mathrm{T}$ cells and production of intracellular IL-4 by $\mathrm{CD}^{+} \mathrm{T}$ cells were significantly inhibited. Furthermore, several reports showed that Th2-related cytokines such as IL-4 and IL-10 were required for the disease pathogenesis of lymphadenopathy and splenomegaly in MRL/lpr mice and human autoimmune lymphoproliferative syndrome (ALPS) patients [14, 37, 39, 44]. Thus, these results suggest that both IL-4 and the CD28/CTLA4-B7 pathway have a role in the propagation of lymphadenopathy and splenomegaly.

It should be noted, however, the development of lymphadenopathy and splenomegaly was also inhibited in IFN- $\gamma$ deficient $l p r$ mice [36]. The critical difference in the profile of cellular subsets between IFN- $\gamma$ deficient $l p r$ and CTLA4IgG-treated $l p r$ mice was that the appearance of DN $T$ cells was almost completely inhibited in IFN- $\gamma$ deficient mice as compared with those of the other cellular subsets [36]. The absence of DN T cells in IFN- $\gamma$ deficient mice clearly shows a requirement for IFN- $\gamma$ in the development of DN T cells. The involvement of IFN- $\gamma$ in the generation of DN T cells is further supported by the fact that there is expansion of DN T cells after injection of IL-12 that induces the augmented expression of IFN- $\gamma$ [45]. Therefore, the generation of DN T cells in MRL/lpr mice appears to be dependent on Fas mutation and IFN- $\gamma$ molecules. The discrepancy on the selective reduction in the number of DN $\mathrm{T}$ cells between IFN- $\gamma$ deficient mice and CTLA4IgG-treated mice can be explained as follows; INF- $\gamma$ production from DN T cells was inhibited by CTLA4IgG, however, INF- $\gamma$ production from macrophages was not suppressed in 

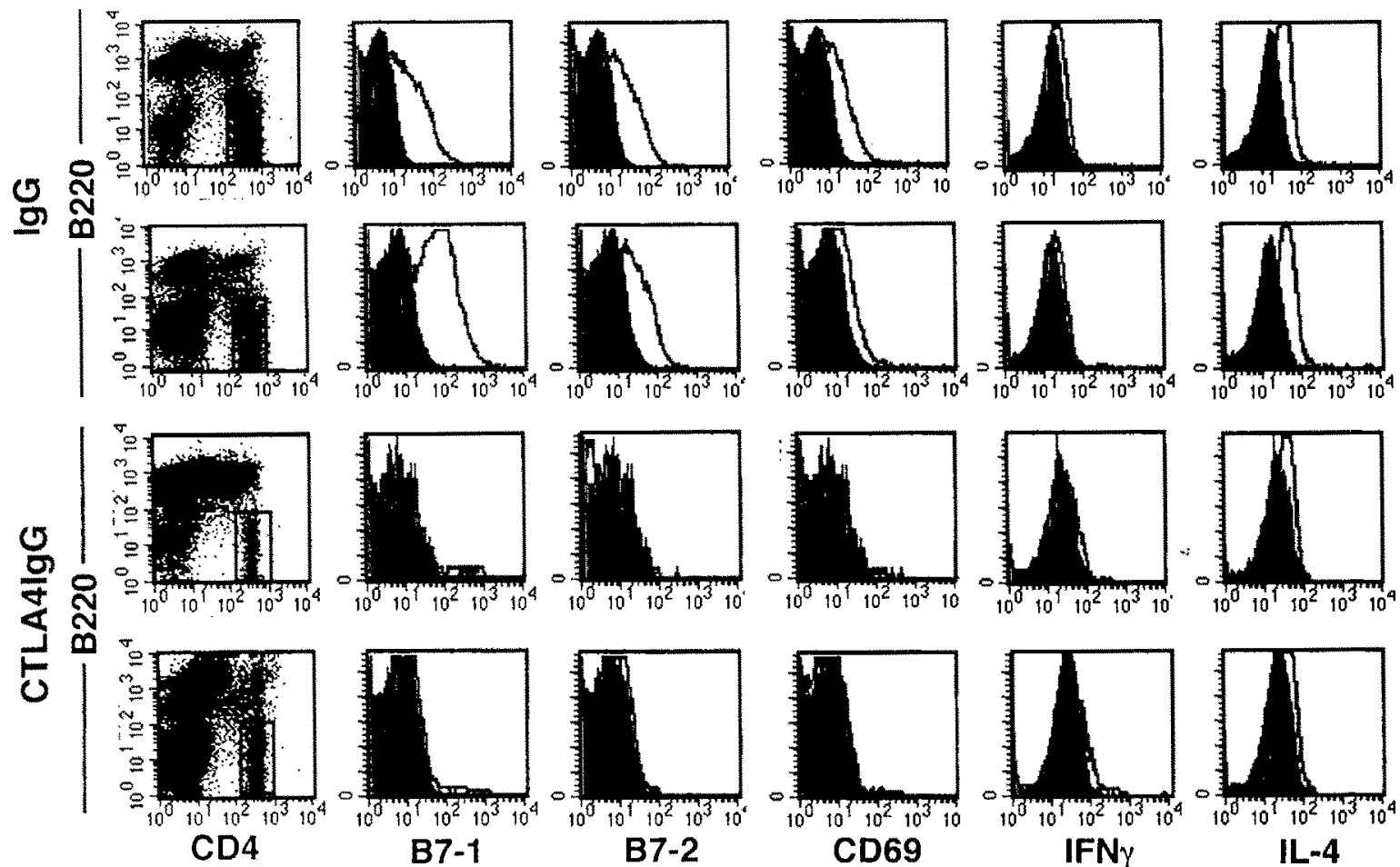

\section{$\frac{\frac{5}{9}}{\frac{8}{0}}$}
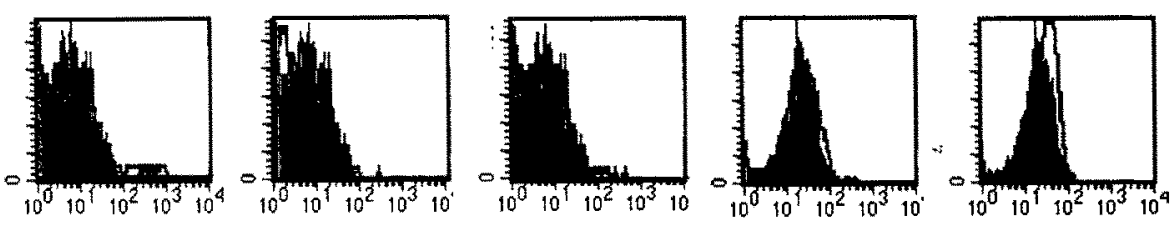

$\mathbf{z}$
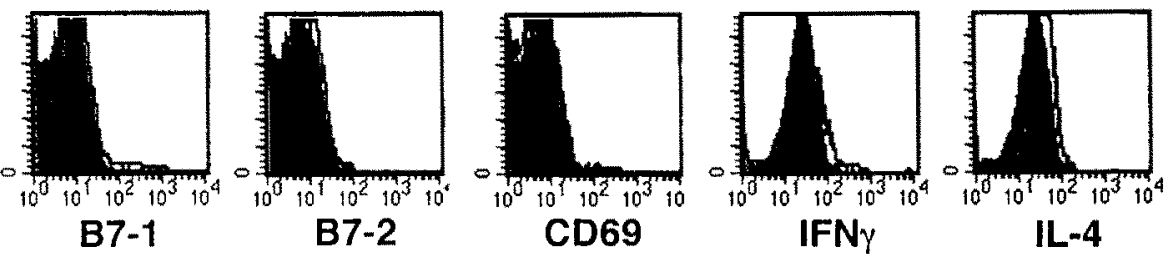

$\frac{\bar{d}}{\text { के }}$

Fig. 4. Activation markers and intracellular cytokines in conventional CD4+ $\mathrm{T}$ cells in MRL/lpr mice. From 20-week-old IgG- or CTLA4IgG-treated MRL/lpr mice, lymph node and spleen cells were harvested. For gating of $\mathrm{CD}^{+} \mathrm{T}$ cells, cells were stained with the combination of anti-B220 and anti -CD4 mAb. The gated cells were then analyzed for surface antigens and intracellular cytokines such as B7-1, B7-2, CD69, IFN- $\gamma$, and IL-4. Experiments were done at least 6 times using different mice and the data were not significantly different. The representative data were shown.
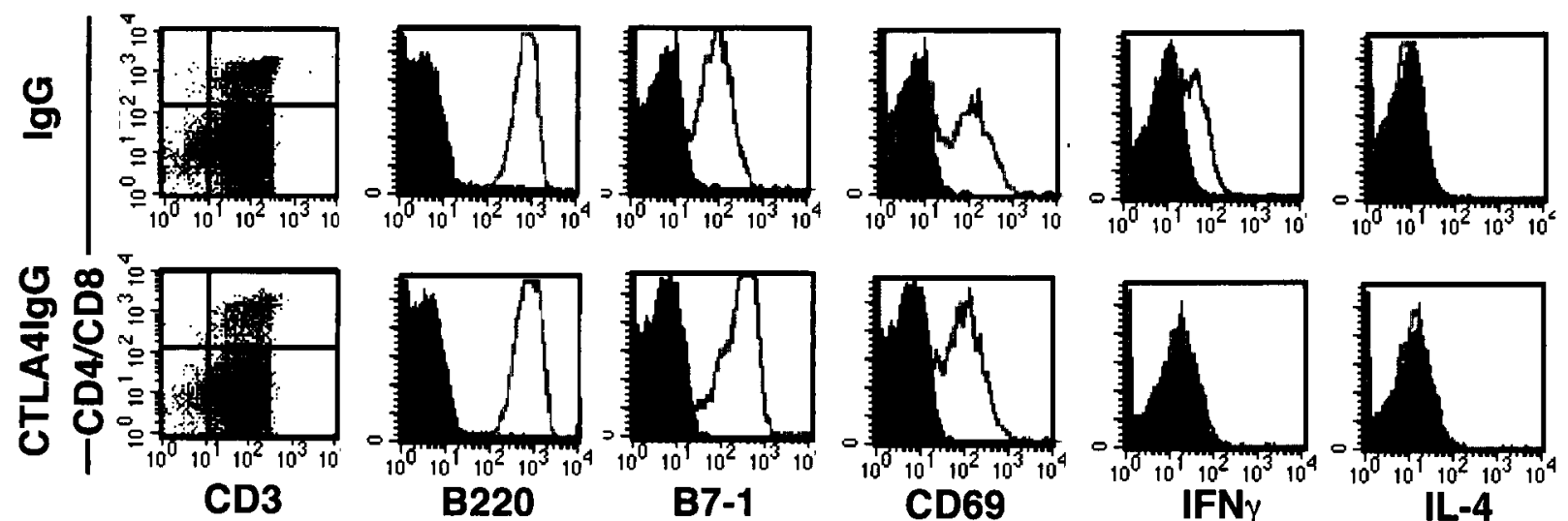

Fig. 5. Activation markers and intracellular cytokine in DN T cells in MRL/lpr mice. From 20-week-old IgG- or CTLA4IgG-treated MRL/lpr mice, lymph node cells were harvested. For gating of DN T cells, cells were stained with anti-CD4 plus anti-CD8 and antiCD3 mAb. The gated cells were then analyzed for surface antigens and intracellular cytokine such as B220, B7-1, CD69, IFN- $\gamma$, and IL-4. Experiments were done at least 6 times using different mice and the data were not significantly different. The representative data were shown.

\section{CTLA4IgG treatment.}

In conclusion, it was demonstrated that (i) the development of DN T cells was independent of the CD28/ CTLA4-B7 pathway, (ii) the CD28/CTLA4-B7 pathway was required for the development of lymphadenopathy and splenomegaly, and (iii) the CD28/CTLA4-B7 signaling pathway in $\mathrm{T}$ cells was important for the accumulation of various cell populations in the lymph node and spleen. Additionally, it was suggested that whereas detected only by the intracellular staining, activated $\mathrm{T}$ cells producing IL- 

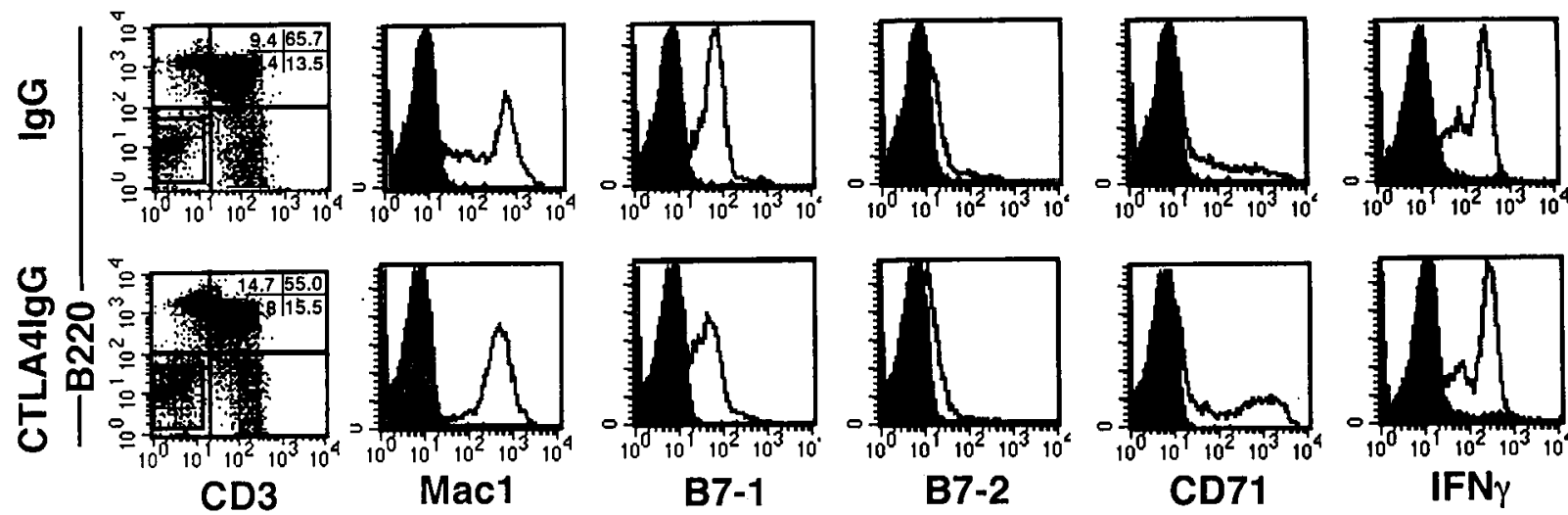

Fig. 6. Activation markers and intracellular cytokine in macrophages in MRL/lpr mice. From 20-week-old IgG- or CTLA4IgGtreated MRL/lpr mice, spleen cells were harvested. For gating of macrophages, cells were stained with the combination of antiB220 and anti-CD3 mAb. The gated cells were then analyzed for surface antigens and intracellular cytokine such as Mac1, B7-1, B7-2, CD71, and IFN- $\gamma$. Experiments were done at least 6 times using different mice and the data were not significantly different. The representative data were shown.

4 may be associated with the propagation of full-blown lymphadenopathy and splenomegaly.

ACKNOWLEDGMENTS. This work was supported in part by a Grant in Aid for Scientific Research on Priority Areas from the Ministry of Education, Science and Culture of Japan, by a research grant from Hokkaido Foundation for The Promotion of Scientific and Industrial Technology, from The Akiyama Foundation, from Kato Memorial Bioscience Foundation, and from Japan Human Sciences Foundation.

\section{REFERENCES}

1. Adachi, M., Watanabe-Fukunaga, R. and Nagata, S. 1993. Aberrant transcription caused by the insertion of an early transposable element in an intron of the Fas antigen gene of lpr mice. Proc. Natl. Acad. Sci. U.S.A. 90: 1756-1760.

2. Allison, J. P. 1994. CD28-B7 interactions in T-cell activation. Curr. Opin. Immunol. 6: 414-419.

3. Andrews, B. S., Eisenberg, R. A., Theofilopoulos, A. N., Izui, S., Wilson, C. B., McConahey, P. J., Murphy, E. D., Roths, J. B. and Dixon, F. J. 1978. Spontaneous murine lupus-like syndromes. Clinical and immunopathological manifestations in several strains. J. Exp. Med. 148: 11981215.

4. Asano, T., Tomooka, S., Serushago, B. A., Himeno, K. and Nomoto, K. 1988. A new T cell subset expressing B220 and CD4 in $l p r$ mice: defects in the response to mitogens and in the production of IL-2. Clin. Exp. Immunol. 74: 36-40.

5. Budd, R. C., Schumacher, J. H., Winslow, G. and Mosmann, T. R. 1991. Elevated production of interferon- $\gamma$ and interleukin 4 by mature $\mathrm{T}$ cells from autoimmune $l p r$ mice correlates with Pgp-1 (CD44) expression. Eur. J. Immunol. 21: 10811084.

6. Chu, E. B., Hobbs, M. V., Wilson, C. B., Romball, C. G., Linsley, P. S. and Weigle, W. O. 1996. Intervention of CD4+ cell subset shifts and autoimmunity in the BXSB mouse by mouse CTLA4Ig. J. Immunol. 156: 1262-1268.

7. Chu, J. L., Drappa, J., Parnassa, A. and Elkon, K. B. 1993. The defect in Fas mRNA expression in MRL/lpr mice is associated with insertion of the retrotransposon, ETn. J. Exp. Med. 178: 723-730.

8. Clements, J. L., Winslow, G., Donahue, C., Cooper, S. M., Allison, J. P. and Budd, R. C. 1993. Co-stimulation via CD28 induces activation of a refractory subset of MRL-lpr/lpr T lymphocytes. Int. Immunol. 5: 1451-1460.

9. Cohen, P. L. and Eisenberg, R. A. 1991. Lpr and gld: single gene models of systemic autoimmunity and lymphoproliferative disease. Annu. Rev. Immunol. 9: 243269.

10. Eisenberg, R. A., Tan, E. M. and Dixon, F. J. 1978. Presence of anti-Sm reactivity in autoimmune mouse strains. J. Exp. Med. 147: 582-587.

11. Finck, B. K., Linsley, P. S. and Wofsy, D. 1994. Treatment of murine lupus with CTLA4Ig. Science 265: 1225-1227.

12. Fisher, G. H., Rosenberg, F. J., Straus, S. E., Dale, J. K., Middleton, L. A., Lin, A. Y., Strober, W., Lenardo, M. J. and Puck, J. M. 1995. Dominant interfering Fas gene mutations impair apoptosis in a human autoimmune lymphoproliferative syndrome. Cell 81: 935-946.

13. Fukuyama, H., Adachi, M., Suematsu, S., Miwa, K., Suda, T., Yoshida, N. and Nagata, S. 1998. Transgenic expression of Fas in T cells blocks lymphoproliferation but not autoimmune disease in MRL-lpr mice. J. Immunol. 160: 3805-3811.

14. Fuss, I. J., Strober, W., Dale, J. K., Fritz, S., Pearlstein, G. R., Puck, J. M., Lenardo, M. J. and Straus, S. E. 1997. Characteristic $\mathrm{T}$ helper $2 \mathrm{~T}$ cell cytokine abnormalities in autoimmune lymphoproliferative syndrome, a syndrome marked by defective apoptosis and humoral autoimmunity. $J$ Immunol. 158: 1912-1918.

15. Giese, T. and Davidson, W. F. 1995. In CD8 ${ }^{+}$T cell-deficient lpr/lpr mice, $\mathrm{CD} 4{ }^{+} \mathrm{B} 220^{+}$and $\mathrm{CD} 4^{+} \mathrm{B} 220^{-} \mathrm{T}$ cells replace $\mathrm{B} 220^{+}$double-negative $\mathrm{T}$ cells as the predominant populations in enlarged lymph nodes. J. Immunol. 154: 4986-4995.

16. Giese, T., Allison, J. P. and Davidson, W. F. 1993. Functionally anergic $l p r$ and $g l d \mathrm{~B} 220^{+} \mathrm{T}$ cell receptor (TCR)- $\alpha / \beta^{+}$ double-negative $\mathrm{T}$ cells express $\mathrm{CD} 28$ and respond to costimulation with phorbol myristate acetate and antibodies to CD28 and the TCR. J. Immunol. 151: 597-609.

17. Inobe, M., Aoki, N., Linsley, P. S., Ledbetter, J. A., Abe, R., Murakami, M. and Uede, T. 1996. The role of the B7-1a 
molecule, an alternatively spliced form of murine B7-1 (CD80), on T cell activation. J. Immunol. 157: 582-588.

18. Inobe, M., Linsley, P. S., Ledbetter, J. A., Nagai, Y., Tamakoshi, M. and Uede, T. 1994. Identification of an alternatively spliced form of the murine homologue of B7. Biochem. Biophys. Res. Commun. 200: 443-449.

19. Jenkins, M. K. and Johnson, J. G. 1993. Molecules involved in T-cell costimulation. Curr. Opin. Immunol. 5: 361-367.

20. June, C. H., Bluestone, J. A., Nadler, L. M. and Thompson, C. B. 1994. The B7 and CD28 receptor families. Immunol. Today 15: 321-332.

21. Kariyone, A., Takiguchi, M., Igarashi, S. and Kano, K. 1988. Ontogeny and function of B220+ $\mathrm{L} 3 \mathrm{~T} 4^{+} \mathrm{T}$-cell subset of MRL/ Mp-lpr/lpr mice. Cell. Immunol. 115: 112-120.

22. Kearney, E. R., Walunas, T. L., Karr, R. W., Morton, P. A., Loh, D. Y., Bluestone, J. A. and Jenkins, M. K. 1995. Antigen-dependent clonal expansion of a trace population of antigen-specific $\mathrm{CD} 4^{+} \mathrm{T}$ cells in vivo is dependent on $\mathrm{CD} 28$ costimulation and inhibited by CTLA-4. J. Immunol. 155: 1032-1036.

23. Kobayashi, S., Hirano, T., Kakinuma, M. and Uede, T. 1993. Transcriptional repression and differential splicing of Fas mRNA by early transposon (ETn) insertion in autoimmune lpr mice. Biochem. Biophys. Res. Commun. 191: 617-624.

24. Kotzin, B. L., Babcock, S. K. and Herron, L. R. 1988. Deletion of potentially self-reactive $\mathrm{T}$ cell receptor specificities in L3T4-, Lyt-2- T cells of lpr mice. J. Exp. Med. 168: 22212229.

25. Krummel, M. F., Sullivan, T. J. and Allison, J. P. 1996. Superantigen responses and co-stimulation: CD28 and CTLA4 have opposing effects on $\mathrm{T}$ cell expansion in vitro and in vivo. Int. Immunol. 8: 519-523.

26. Lafferty, K. J. and Gill, R. G. 1993. The maintenance of selftolerance. Immunol. Cell Biol. 71: 209-214.

27. Leach, D. R., Krummel, M. F. and Allison, J. P. 1996. Enhancement of antitumor immunity by CTLA-4 blockade Science 271(5256): 1734-1736.

28. Liu, Y., Jones, B., Aruffo, A., Sullivan, K. M., Linsley, P. S. and Janeway, C. A. J. 1992. Heat-stable antigen is a costimulatory molecule for CD4 T cell growth. J. Exp. Med. 175: 437-445.

29. Mori, K., Kobayashi, S., Inobe, M., Jia, W. Y., Tamakoshi, M., Miyazaki, T. and Uede, T. 1994. In vivo cytokine gene expression in various $\mathrm{T}$ cell subsets of the autoimmune MRL/ Mp-lpr/lpr mouse. Autoimmunity 17: 49-57.

30. Morse, H. C. I., Davidson, W. F., Yetter, R. A., Murphy, E. D., Roths, J. B. and Coffman, R. L. 1982. Abnormalities induced by the mutant gene lpr: expression of a unique lymphocyte subset. J. Immunol. 129: 2612-2615.

31. Murakami, M., Takahashi, Y., Isashi, Y., Kon, S., Jia, W., Inobe, M., Abe, R. and Uede, T. 1996. Identification and characterization of an alternative cytotoxic $\mathrm{T}$ lymphocyteassociated protein 4 binding molecule on B cells. Proc. Natl. Acad. Sci. U.S.A. 93: 7838-7842.

32. Mountz, J. D., Zhou, T., Eldridge, J., Berry, K. and Bluthmann, H. 1990. Transgenic rearranged $T$ cell receptor gene inhibits lymphadenopathy and accumulation of CD4-CD8-B220+ T cells in lpr/lpr mice. J. Exp. Med. 172: 1805-1817.

33. Murray, L. and Martens, C. 1989. The abnormal T lymphocytes in $l p r$ mice transcribe interferon- $\gamma$ and tumor necrosis factor- $\alpha$ genes spontaneously in vivo. Eur. J. Immunol. 19: $563-565$.

34. Murray, L. J., Lee, R. and Martens, C. 1990. In vivo cytokine gene expression in T cell subsets of the autoimmune MRL/ Mp-lpr/lpr mouse. Eur. J. Immunol. 20: 163-170.

35. Nakagawa, I., Murakami, M., Ijima, K., Chikuma, S., Saito, I., Kanagae, Y., Ishikura, H., Yoshiki, T., Okamoto, H., Kitabatake, A. and Uede, T. 1998. Persistent and secondary adenovirus-mediated hepatic gene expression using adenovector containing CTLA4IgG. Hum. Gene Ther. 9: 1739-1745.

36. Peng, S. L., Moslehi, J. and Craft, J. 1997. Roles of interferon- $\gamma$ and interleukin- 4 in murine lupus. J. Clin. Invest. 99: 1936-1946.

37. Prud'homme, G. J., Kono, D. H. and Theofilopoulos, A. N. 1995. Quantitative polymerase chain reaction analysis reveals marked overexpression of interleukin- $1 \beta$, interleukin-10 and interferon- $\gamma$ mRNA in the lymph nodes of lupus-prone mice. Mol. Immunol. 32: 495-503.

38. Renno, T., Hahne, M., Tschopp, J. and MacDonald, H. R. 1996. Pheripheral T cells undergoing superantigen-induced apoptosis in vivo express B220 and upregulate Fas and Fas lignad. J. Exp. Med. 183: 431-437.

39. Schorlemmer, H. U., Dickneite, G., Kanzy, E. J. and Enssle, K. H. 1995. Modulation of the immunoglobulin dysregulation in GvH- and SLE-like diseases by the murine IL-4 receptor (IL-4-R). Inflamm. Res. 44: S194-S196.

40. Singer, G. G. and Abbas, A. K. 1994. The fas antigen is involved in peripheral but not thymic deletion of T lymphocytes in $\mathrm{T}$ cell receptor transgenic mice. Immunity 1: 365-371.

41. Tarumi, K., Murakami, M., Yagihashi, A., Nakagawa, I., Hirata, K. and Uede, T. 1999. CTLA4IgG treatment induces long-term acceptance of rat small bowel allografts. Transplantation 67: 520-525.

42. Takiguchi, M., Murakami, M., Nakagawa, I., Yamada, A., Chikuma, S., Kawaguchi, Y., Hashimoto, A. and Uede, T. 1999. Blockade of CD28/CTLA4-B7 pathway prevented autoantibody-related diseases but not lung disease in MRL/lpr mice. Lab. Invest. 79: 317-326.

43. Theofilopoulos, A. N. and Dixon, F. J. 1985. Murine models of systemic lupus erythematosus. Adv. Immunol. 37: 269390.

44. Tsai, C. Y., Wu, T. H., Huang, S. F., Sun, K. H., Hsieh, S. C., Han, S. H., Yu, H. S. and Yu, C. L. 1995. Abnormal splenic and thymic IL-4 and TNF- $\alpha$ expression in MRL-lpr/lpr mice. Scand. J. Immunol. 41: 157-163.

45. Tsutsui, T., Mu, J., Ogawa, M., Yu, W. G., Suda, T., Nagaga, S., Saji, F., Murata, Y., Fujiwara, H. and Hamaoka, T. 1997. Administration of IL-12 induces a CD3 ${ }^{+} \mathrm{CD}^{-} \mathrm{CD}^{-} \mathrm{B} 220^{+}$ lymphoid population capable of eliciting cytolysis against Fas-positive tumor cells. J. Immunol. 159: 2599-2605.

46. Van Parijis, L., Ibraghimov, A. and Abbas, A. K. 1996. The roles of costimulation and fas in $\mathrm{T}$ cell apoptosis and peripheral tolerance. Immunity 4: 321-328.

47. Warren, R. W., Caster, S. A., Roths, J. B., Murphy, E. D. and Pisetsky, D. S. 1984. The influence of the $l p r$ gene on B cell activation: differential antibody expression in $l p r$ congenic mouse strains. Clin. Immunol. Immunopathol. 31: 65-77.

48. Watanabe-Fukunaga, R., Brannan, C. I., Copeland, N. G., Jenkins, N. A. and Nagata, S. 1992. Lymphoproliferation disorder in mice explained by defects in Fas antigen that mediates apoptosis. Nature (Lond.) 356: 314-317.

49. Wu, J., Zhou, T., He, J. and Mountz, J. D. 1993. Autoimmune disease in mice due to integration of an endogenous retrovirus in an apoptosis gene. J. Exp. Med. 178: 461-468.

50. Wu, J., Zhou, T., Zhang, J., He, J., Gause, W. C. and Mountz, 
J. D. 1994. Correction of accelerated autoimmune disease by early replacement of the mutated $l p r$ gene with the normal Fas apoptosis gene in the T cells of transgenic MRL-lpr/lpr mice. Proc. Natl. Acad. Sci. U.S.A. 91: 2344-2348.

51. Yamamoto, K., Mori, A., Nakahama, T., Ito, M., Okudaira, H. and Miyamoto, T. 1990. Experimental treatment of au- toimmune MRL-lpr/lpr mice with immunosuppressive compound FK 506. Immunology 69: 222-227.

52. Zhou, T., Bluethmann, H., Eldridge, J., Berry, K. and Mountz, J. D. 1993. Origin of CD4-CD8-B220+ ${ }^{-}$T cells in MRL-lpr/ $l p r$ mice. Clues from a T cell receptor $\beta$ transgenic mouse. $J$. Immunol. 150: 3651-3667. 